\title{
CONVECTIVE INSTABILITIES IN A BACKWARD-FACING STEP FLOW: GLOBAL FORCED PERTURBATIONS
}

\author{
O. Marquet ${ }^{1,2}$ and D. Sipp ${ }^{1}$ \\ ${ }^{1}$ DAFE, ONERA \\ Meudon, France \\ ${ }^{2}$ LadHyx, CNRS-Ecole Polytechnique \\ Palaiseau, France
}

\begin{abstract}
The two-dimensional (2D) stability of a backward-facing step flow is investigated at the low Reynolds number $R e=500$. Optimal energy growth of linear perturbations sustained by harmonic forcing is computed for several frequencies. The maximum sustained energy growth is of order $10^{5}$ at frequency $f_{S} \sim 0.08$ and is related to the excitation of Kelvin-Helmholtz instability in the shear layer of the separated flow. Stability results are compared to a direct numerical simulation (DNS) forced by a random signal.
\end{abstract}

\section{INTRODUCTION}

The instability mechanisms at the origin of the transition to turbulence in separated flows are only partially understood even for low Reynolds number. Whether the transition onset is due to intrinsic or extrinsic dynamics remains an open question. Local stability theory has been used in the past to investigate the linear stability of this flow [1]. However, the assumption of parallelism inherent to this approach is not satisfied in the case of separated flow. Global stability theory, for which two or three inhomogeneous directions are considered, is a promising approach to investigate the stability of nonparallel flows. The classical global stability analysis, based on a temporal modal decomposition of perturbations, is suitable to describe the intrinsic dynamics. The onset of self-sustained perturbations can be then described by a single global mode [2] However, the extrinsic dynamics, defined as the ability of the flow to strongly amplify external perturbations, cannot be captured by a single global mode. A different analysis has to be developed based on the optimization of energy

This is an Open Access article distributed under the terms of the Creative Commons Attribution-Noncommercial License 3.0, which permits unrestricted use, distribution, and reproduction in any noncommercial medium, provided the original work is properly cited. 
perturbation with respect to initial perturbations or external forcing. Two different propositions have recently emerged in the literature. The first one is based on the recognition that global modes are not orthogonal because of the nonnormality of the linearized Navier-Stokes operator. Strong energy growth can thus be achieved by cooperation of global modes. A reduced-order model of the flow dynamics based on global modes is first designed and the optimal response of the reduced-order model to initial perturbations [3] or external forcing [4] is then investigated. The second approach does not reduce the flow dynamics to a finite subspace of global modes but it performs the optimization procedure on the full system. The temporal response of the backward-facing step flow to initial perturbations has recently been investigated in this framework [5, 6]. Strong optimal transient energy growth has been computed and linked to the existence of a strong local convective instability. In the present study, the response of the flow to external forcing is investigated within the second approach. A method is proposed to compute the optimal energy gain between the forcing and the perturbations. Results are compared with those obtained from a nonlinear direct simulation forced with a random signal.

\section{NOISE AMPLIFICATION AND FREQUENCY SELECTION}

A 2D incompressible flow over a backward-facing step of height $h$ is investigated. A Poiseuille flow of maximal velocity $U_{\infty}$ is prescribed at the inlet of the upstream channel, which is also of height $h$. The size of the downstream channel is $2 h$. This flow configuration is the one studied by [2]. All the flow variables are made nondimensional using these quantities. The Reynolds number is defined as Re $=U_{\infty} h / \nu$ where $\nu$ is the kinematic viscosity and is fixed to $\operatorname{Re}=500$ thereinafter. The velocity $\mathbf{u}$ and pressure $p$ fields satisfy the 2D incompressible Navier-Stokes equations. In the present approach, these flow variables are decomposed into a steady component $(\mathbf{U}, P)$, the base-flow, and an unsteady component $\left(\mathbf{u}^{\prime}, p^{\prime}\right)$, the nonlinear perturbation. The base-flow is solution of the steady Navier-Stokes equations:

$$
\begin{aligned}
\mathbf{U} \cdot \nabla \mathbf{U}+\nabla P-\mathrm{Re}^{-1} \nabla^{2} \mathbf{U} & =\mathbf{0} ; \\
\nabla \cdot \mathbf{U} & =0 .
\end{aligned}
$$

The evolution of the nonlinear perturbation onto this base flow is governed by the equations:

$$
\left.\begin{array}{r}
\partial_{t} \mathbf{u}^{\prime}+\mathbf{U} \cdot \nabla \mathbf{u}^{\prime}+\mathbf{u}^{\prime} \cdot \nabla \mathbf{U}+\mathbf{u}^{\prime} \cdot \nabla \mathbf{u}^{\prime}+\nabla p^{\prime}-\operatorname{Re}^{-1} \nabla^{2} \mathbf{u}^{\prime}=\mathbf{f}^{\prime} ; \\
\nabla \cdot \mathbf{u}^{\prime}=0
\end{array}\right\}
$$



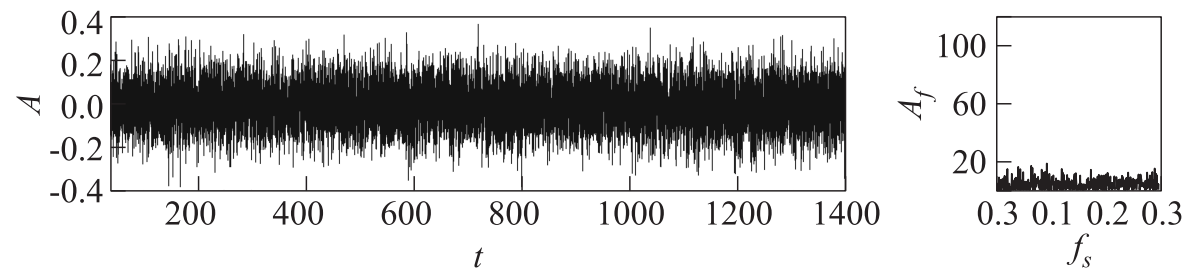

(a)
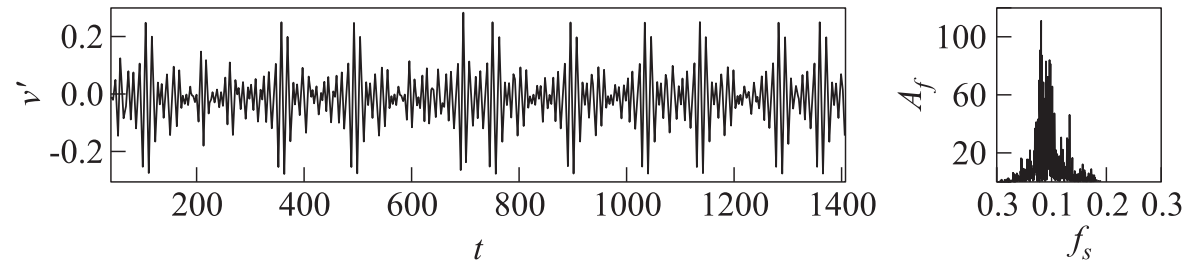

(b)

Figure 1 Time evolution and Fourier spectrum of the forcing amplitude $(a)$ and the vertical velocity perturbation $(b)$ at $x=25, y=-0.5$

where $\mathbf{f}^{\prime}=\left(f^{\prime}, g^{\prime}\right)^{\mathrm{T}}$ is a forcing term $\mathbf{f}^{\prime}=\left(f^{\prime}, g^{\prime}\right)^{\mathrm{T}}$ in the momentum equation that models an external noise. In the present study, it is chosen as a vertical forcing

$$
g^{\prime}(x, y, t)=A\left(t, \mu_{t}, \sigma_{t}\right) \exp \left[-\frac{\left(x-x_{s}\right)^{2}+\left(y-y_{s}\right)^{2}}{2 \sigma_{s}^{2}}\right]
$$

with a Gaussian spatial structure centered at the point $\left(x_{s}, y_{s}\right)$ upstream the backward-facing step. The forcing amplitude $A\left(t, \mu_{t}, \sigma_{t}\right)$ is a time-varying pseudorandom zero-mean $\left(\mu_{t}=0\right)$ Gaussian white-noise signal with standard deviation level $\sigma_{t}=0.1$. The time evolution of the forcing amplitude and its Fourier spectrum are depicted in Fig. $1 a$. Note that the forcing parameters are as follows: $x_{s}=-0.5 ; y_{s}=0.25 ; \sigma_{s}=0.1 ; \mu_{t}=0.0 ;$ and $\sigma_{t}=0.1$

The nonlinear perturbation Eq. (1) are discretized in space onto a finiteelement basis and integrated in time using a second-order semiimplicit scheme. An Uzawa method is used to ensure the incompressibility condition. For more details about the numerical methods, the reader can refer to [7].

The time evolution of the vertical perturbation velocity measured far downstream the backward facing-step $(x=25, y=-0.5)$ and its Fourier spectrum are depicted in Fig. 1b. The spectrum shows a narrow sharp peak with a center frequency $f_{S} \sim 0.08$, very different from the large frequency band of the white-noise external forcing. It is clear that downstream the backward-facing step, only some frequencies of the external forcing are selected by the flow. In the time evolution of the perturbation, one may also note a modulation of the 


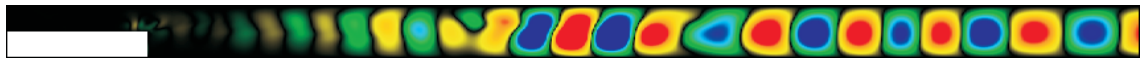

(a)

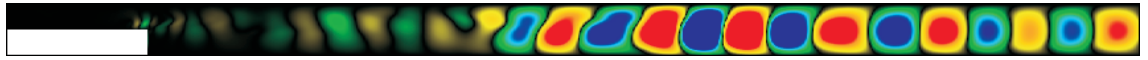

(b)

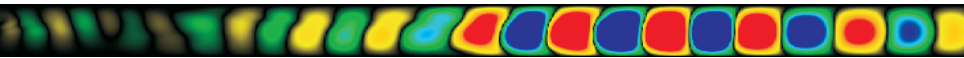

(c)

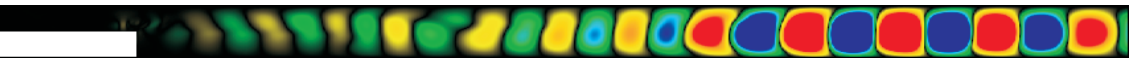

(d)

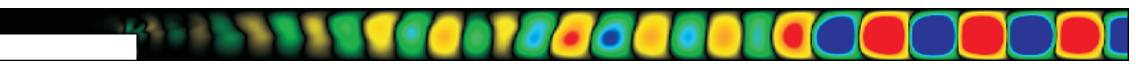

(e)

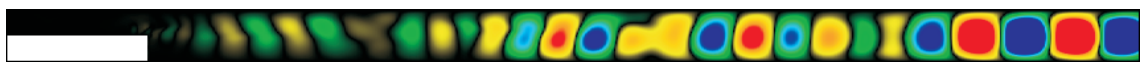

$(f)$

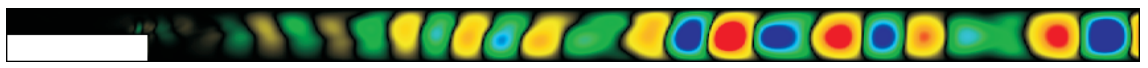

$(g)$

Figure 2 Snapshots of the vertical perturbation for several times of the simulation: (a) $t=80 ; \quad(b) 90 ; \quad(c) 100 ; \quad(d) 110 ; \quad(e) 120 ;(f) 130 ;$ and $(g) t=140 . \quad$ (Refer Marquetand Sipp, p. 454.)

amplitude. This is not related to any low-frequency into the spectrum. In fact, such a time modulation is associated to the narrow shape of the peak spectrum and the existence into the flow of very close frequencies.

Let turn now to the description of the spatial structure existing into the flow. Snapshots of the vertical velocity are depicted in Fig. 2 for several times of the computation. Close to the step, the external forcing introduces very low-amplitude vertical perturbations into the flow. These perturbations are convected downstream and amplified by the shear layer of the first recirculation bubble via a Kelvin-Helmholtz mechanism. While convected downstream, these structures grow in space up to reach the size of the downstream channel. The temporal frequency peak seen in the spectrum (see Fig. 1b) is associated with the convection of these spatial structures.

It is clear from this DNS forced by a random signal that the backward facing step flow amplifies incoming perturbations generated by a white noise external forcing for a narrow frequency band. 


\section{LINEAR FREQUENCY APPROACH}

The global stability of the present flow configuration has been extensively studied in [2]. It was found that, for Reynolds numbers above the critical Reynolds number $\operatorname{Re}_{c} \sim 750$, the base flow is unstable to three-dimensional nonoscillating global modes characterized by spanwise wavenumbers close to 0.9 . This result cannot explain the emergence of unsteadiness in the flow and suggests that the latter is not due to the existence of a self-sustained mechanism but rather to the ability of the flow to amplify external noise. This hypothesis is here investigated by considering the linearized forced Navier-Stokes equations:

$$
\left.\begin{array}{r}
\partial_{t} \mathbf{u}^{\prime}+\mathbf{U} \cdot \nabla \mathbf{u}^{\prime}+\mathbf{u}^{\prime} \cdot \nabla \mathbf{U}+\nabla p^{\prime}-\operatorname{Re}^{-1} \nabla^{2} \mathbf{u}^{\prime}=\mathbf{f}^{\prime} ; \\
\nabla \cdot \mathbf{u}^{\prime}=0 .
\end{array}\right\}
$$

The forcing term is introduced to sustain the linear perturbations $\left(\mathbf{u}^{\prime}, p^{\prime}\right)$ that develop on the base flow $(\mathbf{U}, P)$. It is looked for as an harmonic forcing

$$
\mathbf{f}^{\prime}(x, y, t)=\frac{1}{2}\left\{\mathbf{f}(x, y) e^{\mathrm{i} \omega t}+\text { c.c. }\right\}
$$

characterized by a real forcing pulsation $\omega$ and a complex spatial structure $\mathbf{f}$ $=(f, g)^{\mathrm{T}}$. A similar decomposition is used for the flow perturbations,

$$
\left(\mathbf{u}^{\prime}, p^{\prime}\right)(x, y, t)=\frac{1}{2}\left\{(\mathbf{u}, p)(x, y) e^{\mathrm{i} \omega t}+\text { c.c. }\right\}
$$

where $(\mathbf{u}, p)$ is the spatial structure of the perturbation sustained by the forcing $\mathbf{f}$. By introducing these decompositions into Eqs. (2) yields the relation between the forcing and velocity spatial structures:

$$
\left.\begin{array}{rl}
\mathbf{u} & =\mathcal{R}(\omega) \mathbf{f} \\
\mathcal{R}(\omega) & =\mathcal{P}^{\mathrm{T}}\left(i \omega \mathcal{P} \mathcal{P}^{\mathrm{T}}-\mathcal{L}\right)^{-1} \mathcal{P}
\end{array}\right\}
$$

where $\mathcal{R}(\omega)$ is the resolvant operator; $\mathcal{P}$ is the prolongation operator; and $\mathcal{L}$ is the linearized Navier-Stokes operator. These last two operators are defined by

$$
\mathcal{P}=\left(\begin{array}{c}
\mathbf{I}_{2,2} \\
\mathbf{0}_{1,2}
\end{array}\right) ; \quad \mathcal{L}=\left(\begin{array}{cc}
-\mathbf{U} \cdot \nabla-() \cdot \nabla \mathbf{U}+\mathrm{Re}^{-1} \nabla^{2}-\nabla \\
\nabla \cdot()
\end{array}\right)
$$

with $\nabla=\left(\partial_{x} \partial_{y}\right)^{\mathrm{T}}$. Note that the prolongation operator $\mathcal{P}$ acts on a velocity vector and adds zero pressure components to yield a velocity-pressure vector, while the restriction operator $\mathcal{P}^{\mathrm{T}}$ acts on a velocity-pressure vector and extracts 
the velocity components. By discretizing the continuous problem (3) on a finiteelement basis, one obtains:

$$
\left.\begin{array}{c}
\mathbf{u}=\mathbf{R}(\omega) \mathbf{f} ; \quad \mathbf{R}(\omega)=\mathbf{P}^{\mathrm{T}} \mathbf{S}(\omega)^{-1} \mathbf{P} \mathbf{Q} ; \\
\mathbf{S}(\omega)=i \omega \mathbf{P} \mathbf{Q} \mathbf{P}^{T}-\mathbf{L}
\end{array}\right\}
$$

where the velocity (respectively, forcing) field $\mathbf{u}$ (respectively, $\mathbf{f}$ ) is now a vector containing all the degrees of freedom of the two components of velocity (respectively, forcing); $\mathbf{R}, \mathbf{P}$ and $\mathbf{L}$ are the discrete versions of the continuous operator defined above, and $\mathbf{Q}$ denotes the mass matrix.

The kinetic energy of the perturbation in the domain $\Omega, E(\mathbf{u})=\mathbf{u}^{H} \mathbf{Q u}$ is chosen to measure the growth of the perturbation. The symbol $H$ denotes here the discrete conjugate transpose operation. The energetic gain of the perturbation $\mathbf{u}$ sustained by the forcing $\mathbf{f}$ is given by

$$
G(\omega, \mathbf{f})=\frac{\mathbf{u}^{H} \mathbf{Q u}}{\mathbf{f}^{H} \mathbf{Q f}}=\frac{(\mathbf{R}(\omega) \mathbf{f})^{H} \mathbf{Q R}(\omega) \mathbf{f}}{\mathbf{f}^{H} \mathbf{Q} \mathbf{f}}
$$

where the last part is obtained by considering the relations (4). Introducing $\mathbf{R}^{A}(\omega)$, the adjoint operator of the resolvant (with respect to the discrete energy inner product) which satisfies

$$
\mathbf{u}_{1}^{H} \mathbf{Q R}(\omega) \mathbf{u}_{2}=\left(\mathbf{R}^{A}(\omega) \mathbf{u}_{1}\right)^{H} \mathbf{Q} \mathbf{u}_{2}
$$

for any vectors $\mathbf{u}_{1}$ and $\mathbf{u}_{2}$, the energetic gain can be expressed as a Rayleigh quotient:

$$
\begin{aligned}
& G(\omega, \mathbf{f})=\frac{\left(\mathbf{R}^{A}(\omega) \mathbf{R}(\omega) \mathbf{f}\right)^{H} \mathbf{Q f}}{\mathbf{f}^{H} \mathbf{Q f}} \\
& \mathbf{R}^{A}(\omega)=\mathbf{P}^{T}\left(\mathbf{S}(\omega)^{H}\right)^{-1} \mathbf{P} \mathbf{Q} .
\end{aligned}
$$

Note that the adjoint of the resolvant involves the conjugate transpose of $\mathbf{S}(\omega)$. This Rayleigh quotient can be viewed as the eigenvalue problem:

$$
\mathbf{R}^{A}(\omega) \mathbf{R}(\omega) \hat{\mathbf{f}}_{i}=\lambda_{i}^{2} \hat{\mathbf{f}}_{i} ; \quad \hat{\mathbf{f}}_{i}^{H} \mathbf{Q} \hat{\mathbf{f}}_{i}=1
$$

Since the operator $\mathcal{R}^{H} \mathcal{R}$ is symmetric, the eigenvalues are positive (and denoted $\left.\lambda_{i}^{2}\right)$ and the eigenvectors $\hat{\mathbf{f}}_{i}$ are orthogonal with respect to the energy inner product. The set of eigenvectors $\left\{\hat{\mathbf{f}}_{i}\right\}_{i \geq 1}$ forms, thus, an orthonormal basis of the forcing space $\left(\hat{\mathbf{f}}_{i}, \hat{\mathbf{f}}_{j}\right)=\delta_{i j}$. The normalized optimal responses are defined as

$$
\hat{\mathbf{u}}_{i}=\frac{\mathcal{R} \hat{\mathbf{f}}_{i}}{\lambda_{i}}
$$


It may be shown that $\left(\hat{\mathbf{u}}_{i}, \hat{\mathbf{u}}_{j}\right)=\delta_{i j}$. Also, $\hat{\mathbf{u}}_{i}$ may be obtained through $\mathcal{R} \mathcal{R}^{H} \hat{\mathbf{u}}_{i}=\lambda_{i}^{2} \hat{\mathbf{u}}_{i}$. The eigenvectors $\hat{\mathbf{u}}_{i}$ are orthogonal with respect to the energy inner product. The set of eigenvectors $\left\{\hat{\mathbf{u}}_{i}\right\}_{i \geq 1}$ forms, thus, an orthonormal basis of the response space. For a fixed parameter couple $(\omega, \beta)$, the maximal growth is given by the largest eigenvalue $\lambda_{1}^{2}=\max _{\mathbf{f}} G(\omega, \mathbf{f})$ and the optimal forcing corresponds to the associated eigenvector $\hat{\mathbf{f}}_{1}$.

\subsection{Numerical Method}

The largest eigenvalues of the problem (5) are determined by using the routine znaupd of the ARPACK's library in regular mode. It requires to compute $\mathbf{y}$ $=\mathbf{R}^{A} \mathbf{R} \mathbf{x}$ for some given vector $\mathbf{x}$. This is done in two steps:

(1) $\mathbf{v}=\mathbf{R x}$; and

(2) $\mathbf{y}=\mathbf{R}^{A} \mathbf{v}$.

Each of these steps requires to solve a linear problem. This is achieved by first forming explicitly the appropriate sparse matrices, and then inverting them through a direct LU solver. The MUMPS package is used. Only the matrix $\mathbf{S}$ is stored since this matrix is involved in both inverse. The cost of this algorithm is approximately given by the cost of the $\mathrm{LU}$ decomposition of this large sparse complex matrix. Once this LU decomposition has been achieved, the computational-time cost of the algorithm is cheap.

\subsection{Results}

Figure $3 a$ depicts the optimal energetic gain $\lambda_{1}^{2}$ as a function of the frequency. It shows a sharp peak centered around the frequency $f_{S}=0.075$ where the largest optimal gain is equal to $\lambda_{1}^{2}=5.610^{7}$. This frequency is called the optimal frequency.

In Fig. 3b, a semilogarithmic scale is used to plot the optimal and two first suboptimal energetic gains. At the optimal frequency, the suboptimal gains are four orders of magnitude smaller than the optimal gains. Therefore, the flow perturbation will be dominated by the spatial structure of the optimal perturbation.

The spatial structures of the optimal forcing and perturbation at the optimal frequency are respectively shown in Figs. $4 a$ and $4 b$. The forcing is strongly localized at the step edge and its magnitude decays quickly further downstream. In the shear layer of the lower recirculation bubble, the forcing exhibits structures of opposite sign which scale with the size of the shear layer. It can be concluded that the forcing activate a Kelvin-Helmholtz mechanism in the shear layer of 


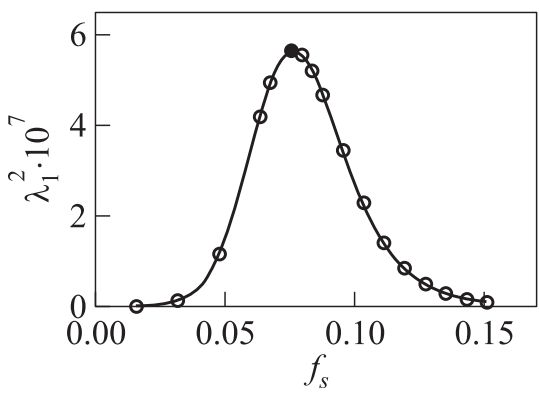

(a)

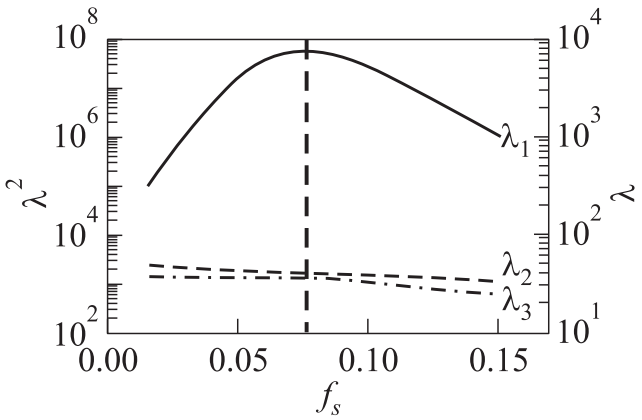

(b)

Figure 3 Optimal energetic gain $\lambda_{1}^{2}$ as a function of the frequency $f_{S}(a)$ and optimal and two suboptimal energetic gains as a function the frequency $(b)$; semilogarithmic scale
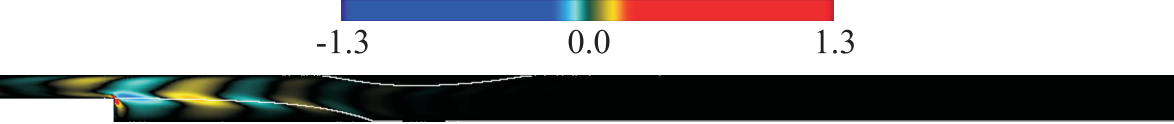

(a)

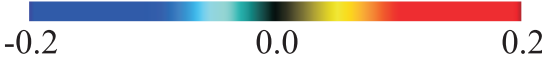

(b)

Figure 4 Spatial structure of the forcing $(a)$ and the perturbation $(b)$ associated to the optimal energetic gain $\lambda_{1}^{2}$ at frequency $f_{S}=0.075$ (real part of the vertical component). (Refer Marquet and Sipp, p. 458.)

the recirculation bubble. Moreover, it can be noted that these structures are inclined in the opposite direction to the shear of the base flow. This also suggests that an Orr mechanism is active and contributes to the energetic amplification. Compared to the forcing, the perturbation is located further downstream and is much less localized. Its maximal magnitude is reached at the station $x \sim 25$. The spatial structures are of opposite sign and scale with the downstream channel height 2. The imaginary part of the vertical velocity (not shown here) is in quadrature of phase with the real part. This indicates that the perturbation is a travelling wave with phase speed equal to $\omega / 2=0.237$. The spatial separation of the forcing and perturbation structures can be related to the convective nonnormality of the linearized Navier-Stokes operator. 


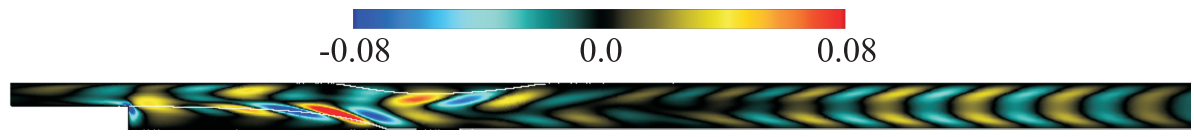

(a)

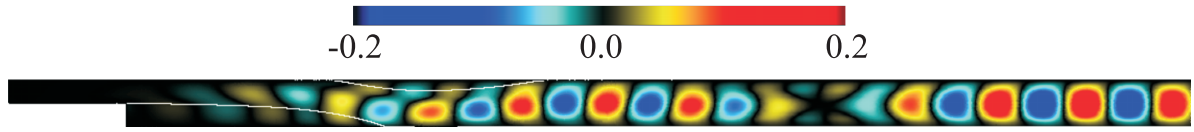

(b)

Figure 5 The same as in Fig. 4 but for the the first suboptimal energetic gain $\lambda_{2}^{2}$. (Refer Marquet and Sipp, p.459.)

The spatial structure of the forcing and perturbation of the first suboptimal is shown in Figs. $5 a$ and $5 b$. The suboptimal forcing exhibits a very different pattern compared to the optimal forcing. It is not localized at the step edge but distributed in the whole domain. The largest magnitudes are obtained in the downstream channel $(x \sim 25)$, in the downstream part of the lower recirculation bubble, and in the shear layer of the upper recirculation bubbles. In the downstream channel, where the base flow progressively recovers a Poiseuille flow, the forcing structure is very similar to the optimal forcing structure in a Poiseuille flow. The spatial structure of the suboptimal perturbation is very similar to the one of the optimal perturbation, except that it is strongly modulated in space. Indeed, its magnitude almost vanishes at the station $x=25$ where the forcing is maximal. It is interesting to note that the forcing and perturbation are not separated in space as for the optimal structures.

\subsection{Comparison}

Results obtained with the present stability analysis show good agreement with results of the forced DNS. The energetic gain as a function of the frequency (see Fig. 3) shows strong similarity with the Fourier spectrum plotted in Fig. 1. In particular, the optimal frequency $f_{S}=0.075$ is close to the frequency where the Fourier spectrum exhibits a peak $f_{S}=0.08$. The correspondence between the spatial structures of the perturbation (see Fig. 4) and of the snapshots (see Fig. 2) is also good in the downstream channel.

\section{REFERENCES}

1. Kaiktsis, L., G. E. Karniadakis, and S. A. Orszag. 1996. Unsteadiness and convective instabilites in two-dimensional flow over a backward-facing step. J. Fluid Mech., 321:157-87. 
2. Barkley, D., M. Gomes, and D. Henderson. 2002. Three-dimensional instability in flow over a backward-facing step. J. Fluid Mech. 473:167-89.

3. Ehrenstein, U., and F. Gallaire. 2005. On two-dimensional temproal modes in spatially evolving open flows: The flat-plate boundary layer. J. Fluid Mech. 536:209-18.

4. Alizard, F., S. Cherubini, and J.-C. Robinet. 2009. Sensitivity and optimal forcing response in separated boundary layer flows. Phys. Fluids 21:064108.

5. Blackburn, H. M. D. Barkley, and S. J. Sherwin. 2008. Convective instability and transient growth in flow over a backward-facing step. J. Fluid Mech. 603:271-304.

6. Marquet, O., D. Sipp, J. M. Chomaz, and L. Jacquin. 2008. Amplifier and resonator dynamics of a low-Reynolds-number recirculation bubble in a global framework. J. Fluid Mech. 605:429-43.

7. Sipp, D., and A. Lebedev. 2007. Global stability of base- and mean-flows: A general approach and its applications to cylinder and open cavity flows. J. Fluid Mech. 593:333-58. 